\title{
La metformina reduciría la incidencia de diabetes mellitus tipo 2 en personas con mayor riesgo de desarrollar esta enfermedad
}

Metformin may reduce the incidence of type 2 diabetes mellitus in persons at increased risk for the development of this condition

\section{Comentado de:}

Madsen KS, et al. Cochrane Database of Systematic Reviews 2019, Issue 12. Art. No.: CD008558. PMID: $31794067^{1}$

\section{Introducción}

El aumento proyectado en la incidencia de diabetes mellitus tipo 2 (DM2) se podría convertir en un problema significativo de salud en todo el mundo. Se desconoce si la metformina puede prevenir o retrasar la DM2 y sus complicaciones en personas con mayor riesgo de desarrollar esta enfermedad (intolerancia a la glucosa o glucemia alterada en ayunas).

\section{Objetivos}

Evaluar los efectos de la metformina para la prevención o el retraso de la DM2 y sus complicaciones asociadas en personas con mayor riesgo de desarrollar DM2.

\section{Métodos de búsqueda}

Se hicieron búsquedas en el Registro Cochrane Central de Ensayos Controlados (Cochrane Central Register of Controlled Trials), MEDLINE, Scopus, ClinicalTrials.gov, la plataforma International Clinical Trials Registry Platform de la Organización Mundial de la Salud (OMS), y en las listas de referencias de revisiones sistemáticas, artículos e informes de evaluación de tecnología sanitaria. Se solicitó a los investigadores de los ensayos incluidos información acerca de ensayos adicionales. La fecha de la última búsqueda para todas las bases de datos fue marzo de 2019.

\section{Criterios de selección}

Se incluyeron ensayos controlados aleatorizados (ECA) con una duración de un año o más que compararan la metformina con cualquier intervención farmacológica para reducción de la glucosa, alguna intervención de cambio de conducta, placebo o la atención estándar en personas con intolerancia a la glucosa, glucemia alterada en ayunas, hemoglobina glucosilada $\mathrm{A} 1 \mathrm{c}(\mathrm{HbA} 1 \mathrm{c})$ moderadamente elevada o combinaciones de los anteriores.

\section{Obtención y análisis de los datos}

Dos revisores independientes leyeron todos los resúmenes y textos completos, evaluaron el riesgo de sesgo y extrajeron los datos de los resultados. Se utilizó un modelo de efectos aleatorios para realizar el meta-análisis, y se calcularon los riesgos relativos (RR), para los resultados dicotómicos, y las diferencias de medias (DM), para los resultados continuos, con sus intervalos de confianza del $95 \%$ (IC95\%) para las estimaciones del efecto. La certeza de la evidencia se evaluó con criterios GRADE.

\section{Resultados principales}

Se incluyeron 20 ECA que aleatorizaron a 6.774 participantes. Un ensayo aportó $48 \%$ del total de participantes. La duración de la intervención en los ensayos varió de uno a cinco años. Ninguno de los ensayos tuvo bajo riesgo de sesgo en todos los dominios evaluados.

Las principales medidas de resultado fueron mortalidad por todas las causas, incidencia de DM2, eventos adversos graves
(EAG), mortalidad cardiovascular, infarto de miocardio no mortal o accidente cerebrovascular, calidad de vida relacionada con la salud y efectos socioeconómicos.

Las siguientes comparaciones informaron, en su mayoría, solo una fracción del conjunto de resultados principales.

Metformina vs. dieta y ejercicio con o sin placebo (15 ensayos)

- La mortalidad por todas las causas fue de $7 / 1.353$ vs. 7/1.480 (RR 1,11; IC95\%: 0,41 a 3,01; $p=0,83 ; 2833$ participantes, cinco ensayos; evidencia de muy baja calidad).

- La incidencia de DM2 fue de 324/1.751 vs.529/1.881 participantes (RR 0,50; IC95\%: 0,38 a 0,65; p < 0,001; 3632 participantes, 12 ensayos; evidencia de moderada calidad).

- No se pudo realizar el meta-análisis de EAG (los números informados fueron 4/118 vs. 2/191; 309 participantes; cuatro ensayos; evidencia de muy baja calidad)

- La mortalidad cardiovascular fue de 1/1.073 vs.4/1.082 (2.416 participantes; dos ensayos; evidencia de muy baja calidad).

- Un ensayo no informó diferencias claras en la calidad de vida relacionada con la salud después de 3,2 años de seguimiento (evidencia de muy baja calidad).

- Dos ensayos estimaron los costos médicos directos (CMD) por participante, para la metformina variaron entre US\$220 y US $\$ 1.177$ vs. US $\$ 61$ a US $\$ 184$ en el grupo de comparación (2.416 participantes; evidencia de baja calidad).

Metformina vs. dieta intensiva y ejercicio (ocho ensayos)

- La mortalidad por todas las causas fue de $7 / 1.278$ vs.4/1.272 (RR 1,61; IC95\%: 0,50 a 5,23; p = 0,43; 2.550 participantes, cuatro ensayos; evidencia de muy baja calidad).

- La incidencia de DM2 fue de 304/1.455 vs. 251/1.505 (RR 0,80; IC95\%: 0,47 a 1,37; $p=0,42 ; 2.960$ participantes, siete ensayos; evidencia de moderada calidad).

- No se pudo realizar el meta-análisis de EAG (un ensayo informó 1/44 en el grupo de metformina vs. 0/36 en el grupo de ejercicio intensivo y dieta con EAG).

- Un ensayo informó que 1/1.073 participantes del grupo de metformina, vs. 2/1.079 participantes del grupo de comparación, murieron por causas cardiovasculares, mientras que otro estudio informó que ningún participante murió debido a causas cardiovasculares (evidencia de muy baja calidad).

- Dos ensayos estimaron los CMD por participante, para la metformina variaron entre US\$220 y US $\$ 1.177$ vs. US $\$ 225$ a US $\$ 3.628$ en el grupo de comparación (2.400 participantes; evidencia de muy baja calidad). 


\section{Metformina más dieta intensiva y ejercicio vs. dieta intensiva y ejercicio idénticos (tres ensayos)}

- La mortalidad por todas las causas fue 1/121 vs. 1/120 participantes (450 participantes; dos ensayos; evidencia de muy baja calidad)

- La incidencia de DM2 fue de 48/166 vs. 53/166 (RR 0,55 IC95 \%: 0,10 a 2,92; $p=0,49$; 332 participantes; dos ensayos; evidencia de muy baja calidad).

- Un ensayo estimó los CMD por participante, para la metformina más dieta y ejercicio fueron de US\$270 vs. US\$225 en el grupo de comparación (94 participantes; evidencia de muy baja calidad).

\section{Metformina vs. acarbosa (tres ensayos)}

- La mortalidad por todas las causas fue 1/44 vs. 0/45 (89 participantes; un ensayo; evidencia de muy baja calidad).

- La incidencia de DM2 fue 12/147 vs. 7/148 (RR 1,72, IC $95 \% 0,72$ a 4,14; $p=0,22 ; 295$ participantes; tres ensayos; evidencia de baja calidad).

- Los EAG fueron 1/51 vs. 2/50 (101 participantes; un ensayo; evidencia de muy baja calidad).

\section{Metformina vs. tiazolidinedionas (tres ensayos)}

- La incidencia de DM2 fue 9/161 vs. 9/159 (RR 0,99, IC $95 \%$ 0,41 a 2,40; $p=0,98 ; 320$ participantes; tres ensayos; evidencia de baja calidad).
- Los EAG fueron 3/45 vs. 0/41 (86 participantes; un ensayo; evidencia de muy baja calidad).

\section{Metformina vs. sulfonilureas}

- Un ensayo de 45 participantes que realizó esta comparación no informó resultados importantes para los pacientes.

En niguna de las comparaciones hubo datos sobre infarto de miocardio no fatal, accidente cerebrovascular no fatal o complicaciones microvasculares.

\section{Conclusiones}

Evidencia de moderada calidad mostró que la metformina, comparada con placebo o dieta y ejercicio, redujo o retrasó el riesgo de DM2 en personas con mayor riesgo de desarrollarla, pero no fue así cuando se la comparó con dieta intensiva y ejercicio. De igual manera, la adición de metformina a una dieta intensiva y ejercicio no mostró ventajas ni desventajas con respecto al desarrollo de DM2 (evidencia de muy baja calidad). Los datos sobre los resultados importantes para los pacientes, como mortalidad, complicaciones diabéticas macro y microvasculares y calidad de vida relacionada con la salud, fueron escasos o inexistentes.

Fuente de financiamiento/Conflicto de interés de los autores:: Esta revisión sistemática fue financiada por el Hospital West China de la Universidad Sichuan, China. Los autores declararon no tener conflictos de interés.

\section{Comentario}

Esta revisión Cochrane ${ }^{1}$ muestra que la metformina probablemente reduzca o retrase el desarrollo de DM2 en un $50 \%$, comparada con placebo o dieta no intensiva y ejercicio. Tras uno a cinco años de seguimiento, 140 pacientes menos (IC95\%: 98 a 174) cada 1000 pacientes tratados con metformina, desarrollarán DM2. Sin embargo, la metformina probablemente carezca de efecto cuando un paciente además del ejercicio realiza una dieta intensiva.

La evidencia sobre mortalidad, complicaciones macro y microvasculares y calidad de vida fue insuficiente para todas las comparaciones. Los costos médicos directos con metformina fueron mayores que con el tratamiento no farmacológico, aunque hubo diferencias entre ensayos y en los distintos ámbitos de aplicación.

En el mayor ensayo del meta-análisis, el Programa de Prevención de Diabetes de pacientes obesos con intolerancia a la glucosa, la metformina redujo la tasa de progresión a diabetes en comparación con el placebo a los tres años, pero este efecto fue aun más marcado con cambios intensivos de estilo de vida. La incidencia de diabetes fue aproximadamente de 11, 8 y 5 casos por cada 100 personas-año en los grupos de placebo, metformina y estilo de vida, respectivamente. La reducción de la incidencia de DM2 fue de $58 \%$ con cambios en el estilo de vida y $31 \%$ con metformina, en comparación con el placebo. Para prevenir un caso de diabetes durante un período de tres años, 7 personas tendrían que participar en el programa de intervención de estilo de vida, y 14 tendrían que recibir metformina.

En cuanto a las limitaciones de la evidencia resumida, cabe destacar que todos los ensayos tuvieron riesgo de sesgo incierto o alto en uno o más dominios evaluados. Los ECA utilizaron distintas dosis de metformina y, además, algunos estudios utilizaron definiciones no estándar de hiperglucemia.

Un dato no menor es que en los grupos de control de los ensayos incluidos se detectaron más casos incidentes de DM2 que en ensayos observacionales por un control más estricto que el del "mundo real"2,3.

Varias revisiones sistemáticas han investigado estrategias para prevenir o retrasar la DM2 en personas con mayor riesgo de desarrollarla, pero pocas se centraron en la metformina para la prevención de la DM2. Una revisión sistemática que incluyó tres ECA con un tiempo de seguimiento de al menos seis meses encontró que la metformina redujo la incidencia de DM2 alrededor de $35 \%$ en personas con intolerancia a la glucosa o glucemia alterada en ayunas ${ }^{4}$. Otra incluyó 31 ECA con una duración de al menos ocho semanas ${ }^{5}$, con personas con mayor riesgo de DM2, definidas como personas con obesidad, síndrome de ovario poliquístico, resistencia a la insulina, intolerancia a la glucosa, antecedentes familiares de diabetes, enfermedad vascular periférica o síndrome metabólico. Encontró una reducción de $40 \%$ la incidencia de DM2; sin embargo dado sus amplios criterios de inclusión, incluyendo personas normoglucémicas, no resulta muy comparable.

Se ha cuestionado que el beneficio de la metformina en la prevención de la diabetes podría representar sólo un retraso en el desarrollo de la DM2 en lugar de una verdadera prevención, ya que, en la mayoría de los estudios, las pruebas de tolerancia oral a la glucosa de seguimiento se realizaron mientras los pacientes todavía tomaban la metformina. En un estudio de seguimiento de 1.274 sujetos en el grupo de metformina que no habían desarrollado diabetes, las pruebas de tolerancia después de suspender la metformina (en promedio, 11 días) mostraron que aproximadamente el $75 \%$ del beneficio de metformina persistió. ${ }^{6}$ Aunque este hallazgo es consistente con la prevención de nuevos casos, se necesitan seguimientos 
más largos, sin medicamentos, para arrojar conclusiones más firmes al respecto.

La guía más reciente y específica sobre prevención primaria de enfermedad cardiovascular ateroesclerótica y DM2 en pacientes con riesgo metabólico recomienda en personas con prediabetes y limitaciones para la actividad física o que no responden a las modificaciones del estilo de vida, a la metformina como primera línea farmacológica para reducir los niveles de glucosa en plasma ${ }^{7}$. En el mismo sentido, la guía nacional de diabetes de Argentina, en su fase final de desarrollo, parece avanzar en un consenso para considerar apropiado que en personas con tolerancia a la glucosa alterada o glucemia alterada en ayunas que ya implementan un programa intensivo de estilo de vida saludable, pero que continúan deteriorando sus valores glucémicos en los siguientes 3 a 6 meses, o bien, en quienes no adhieren a su adopción, se sugiera iniciar la terapia con metformina (500 a $1700 \mathrm{mg}$ por día), para reducir la incidencia de desarrollo de DM2, salvo que se detecten contraindicaciones para recibir este medicamento ${ }^{8}$.

\section{Conclusiones del comentador}

Tanto los cambios intensivos en el estilo de vida como el tratamiento con metformina reducen la incidencia de diabetes en personas de alto riesgo de desarrollarla. La intervención en el estilo de vida podría ser incluso más efectiva que la metformina, por lo que el abordaje inicial más apropiado sería intentar estos cambios intensivamente, y sólo si no son adoptados (por negativa, inaccesibilidad u otras razones) sería razonable iniciar metformina con el objetivo de reducir o retrasar la DM2. Lamentablemente, la evidencia es insuficiente como para saber si esto podría reflejarse en resultados más importantes para los pacientes, como reducciones de la mortalidad, las complicaciones macro y microvasculares y mejoras en la calidad de vida relacionada con la salud.

Agustin Ciapponi [ Servicio de Medicina Familiar y Comunitaria, Hospital Italiano de Buenos Aires; Centro Cochrane Argentina, Instituto de Efectividad Clínica y Sanitaria. aciapponi@iecs.org.ar ]

Ciapponi A. La metformina reduciría la incidencia de diabetes mellitus tipo 2 en personas con mayor riesgo de desarrollar esta enfermedad. Evid Actual Pract Ambul. 2020;23(1):e002041. Comentado de: Madsen KS, et al. Metformin for prevention or delay of type 2 diabetes mellitus and its associated complications in persons at increased risk for the development of type 2 diabetes mellitus. Cochrane Database of Systematic Reviews 2019 , Issue 12. Art. No.: CD008558. PMID: 31794067

\section{Referencias}

1. Madsen KS, Metzendorf CY, Richter MI, et al. Metformin for prevention or delay of type 2 diabetes mellitus and its associated complications in persons at increased risk for the development of type 2 diabetes mellitus. Cochrane Database Syst Rev. 2019;12:8558-8558. Available from: 10.1002/14651858.CD008558.pub2.

2. Cheng $\mathrm{C}$, Kushner $\mathrm{H}$, Falkner BE. The utility of fasting glucose for detection of prediabetes. Metabolism. 2006;55(4):434-438. Available from 10.1016/j.metabol.2005.10.003.

3. Morris $\mathrm{DH}$, Khunti $\mathrm{K}$, Achana F, et al. Progression rates from $\mathrm{HbA1c} 6.0-6.4 \%$ and other prediabetes definitions to type 2 diabetes: a meta-analysis Diabetologia. 2013;56(7):1489-1493. Available from: 10.1007/s00125-013-2902-4

4. Lily M, Godwin M. Treating prediabetes with metformin: systematic review and meta-analysis. Can Fam Physician. 2009;55(4):363-369.

5. Salpeter SR, Buckley NS, Kahn JA, et al. Meta-analysis: metformin treatment in persons at risk for diabetes mellitus. Am J Med. 2008;121(2):149157. Available from: 10.1016/j.amjmed.2007.09.016.

6. Diabetes Prevention Program Research Group. Effects of withdrawal from metformin on the development of diabetes in the diabetes prevention program. Diabetes Care. 2003;26(4):977-980. Available from: 10.2337/diacare.26.4.977.

7. Rosenzweig JL, Bakris GL, Berglund LF, et al. Primary Prevention of ASCVD and T2DM in Patients at Metabolic Risk: An Endocrine Society* Clinica Practice Guideline. J Clin Endocrinol Metab. 2019;Available from: 10.1210/jc.2019-01338.

8. Salud avanza en el consenso para la nueva guía de práctica clínica de diabetes tipo 2. Argentina;. Published 2019. Accessed 28/12/2019. Available from: http:/www.msal.gob.ar/ent/index.php?option=com_content\&view=article\&id=606:salud-avanza-en-el-consenso-para-la-nueva-guia-depractica-clinica-de-diabetes-tipo-2\&catid=6:destacados-slide 606 . 